\section{Future looks bleak for powerless Hubble device}

Tony Reichhardt, Washington

One of the Hubble Space Telescope's four scientific instruments has shut down and seems unlikely to be resurrected.

Astronomers are not optimistic about repairing the Space Telescope Imaging Spectrograph, one of the telescope's workhorses. "There's some hope, but it is small," says Bruce Margon, a science director at the Space Telescope Science Institute in Baltimore, Maryland.

The spectrograph, which records specific frequencies of visible and ultraviolet light, failed on 3 August

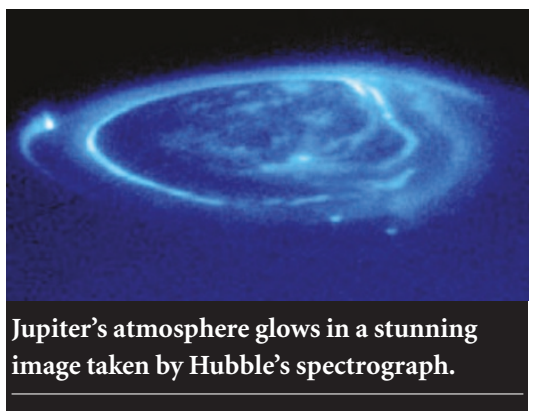

owing to a faulty power unit. Project officials are currently mulling over the few options open to them. The instrument has a back-up power supply, but that has not been used since it was repaired in March 2002 and engineers are not sure whether it will work properly.

The instrument has already lasted two years longer that it was designed to do. Astronomers had come to rely on it: at present, it features in some $30 \%$ of planned Hubble science observations.

If the device cannot be revived, the onboard Advanced Camera for Surveys could provide limited spectra images. Hubble's data could also be supplemented with spectra taken from the ground.

The Cosmic Origins Spectrograph, due to be installed on Hubble during a repair mission in 2006, could perform a few of the imaging spectrograph's functions. But NASA is currently reviewing plans for the 2006 repairs and deciding whether astronauts or robots should do the job, or whether it should be cancelled altogether. The agency said this week that it would start developing plans for a robotic mission, but would not make its final decision until next year.

NASA has also awarded a study contract to a team at the Johns Hopkins University in Baltimore to see if the Cosmic Origins Spectrograph and another camera planned for Hubble could fly on a separate satellite instead.

\section{Fears grow as blood stocks pass on prions undetected}

Michael Hopkin, London

New infections of variant Creutzfeldt-Jakob disease (vCJD) could continue unchecked unless a sensitive screen for blood donors can be devised, researchers have warned.

Most of the $142 \mathrm{vCJD}$ deaths in Britain are believed to have been caused by people eating beef products infected with bovine spongiform encephalopathy. But two people are now known to have been infected through blood transfusions containing disease-causing proteins known as prions. Researchers are concerned that in the latest case, revealed last week, the recipient of the transfusion carried the prions for years without developing the disease.

If unwitting carriers are giving blood, the spread of the disease could be perpetuated even though almost all infected beef has now been removed from the food chain. "That is a worst-case scenario, but we cannot rule it out," says James Ironside of the National Creutzfeldt-Jakob Disease Surveillance Unit in Edinburgh.

Ironside and his colleagues discovered rogue prions during an autopsy of a seemingly disease-free subject who had received a blood transfusion in 1999. They published their results on 6 August (see A. H. Peden, M. W. Head, D. L. Ritchie and J. W. Ironside Lancet 364, 527-529; 2004). The elderly patient, who died of an aneurysm, had small lymph nodes. The patient is the second person in Britain known to have been infected with disease-causing prions through a blood transfusion, but the subject in the first case went on to develop vCJD. amounts of the protein in the spleen and
Researchers doubt whether current precautions, such as stripping donated blood of the white blood cells in which prions are thought to amass, are sufficient to prevent prions from being passed on by transfusions. The UK government also prohibits people who have had a blood transfusion from giving blood, but this would not prevent prions from being passed on by people infected by beef products.

All previous vCJD cases have occurred in people of a particular genetic type, but Ironside's patient belonged to a different group. Adriano Aguzzi, a neuropathologist at the University Hospital of Zurich in Switzerland, speculates that some genetic groups may act as carriers of the infectious protein without developing symptoms themselves. It will be important to see whether the rate of these infections is sufficient to maintain the epidemic, he adds.

Many more patients could contract the disease before we are able to estimate incubation periods, warns Moira Bruce, who studies prion diseases at the Institute for Animal Health in Edinburgh. Ultimately, a sensitive screen for prions in blood samples is needed, she says: "One of the holy grails is an antibody that can distinguish between normal and disease-causing prions." Bruce believes work in this area could bear fruit in the next few years.

In the meantime, says Ironside, we should be aware of the trade-off between the risks and the benefits of blood donations. "Blood is given for good reasons," he points out. "In many cases, if people do not get blood transfusions they die."

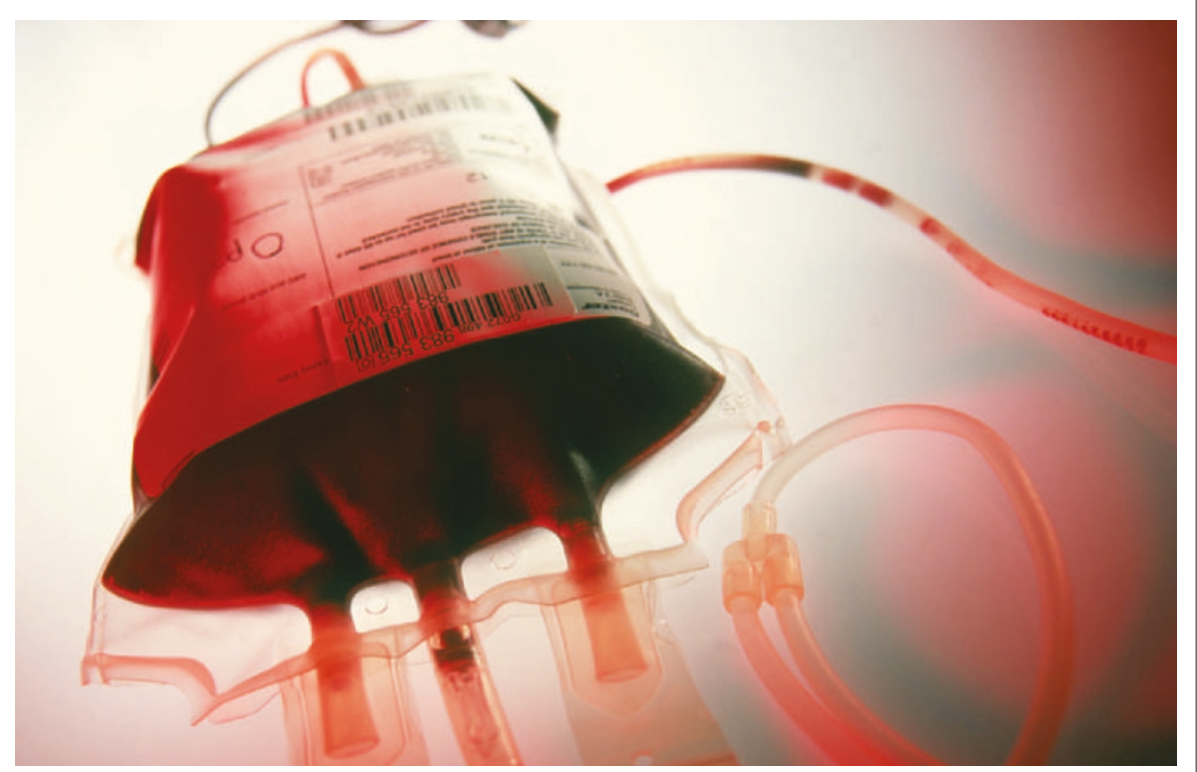

In the blood: donors of certain genetic types may incubate vCJD and pass it on while seeming healthy. 